\title{
Allopregnanolone and dehydroepiandrosterone response to corticotropin-releasing factor in patients suffering from Alzheimer's disease and vascular dementia
}

\author{
Francesca Bernardi, Antonio Lanzone ${ }^{1}$, Rosa M Cento ${ }^{1}$, Rosario S Spada ${ }^{1}$, Ilaria Pezzani ${ }^{2}$, Alessandro David \\ Genazzani $^{3}$, Stefano Luisi, Michele Luisi ${ }^{4}$, Felice Petraglia ${ }^{2}$ and Andrea Riccardo Genazzani \\ Department of Reproductive Medicine and Child Development, Section of Gynecology and Obstetrics, University of Pisa, Italy, ${ }^{1}$ Department of \\ Obstetrics and Gynecology, Oasi, Troina, Italy, ${ }^{2}$ Department of Surgical Science, Chair of Obstetrics and Gynecology, University of Udine, Italy, \\ ${ }^{3}$ Institute of Obstetrics and Gynecology, University of Modena, Italy and ${ }^{4} \mathrm{CNR}$, Endocrine Research Unit, Pisa, Italy \\ (Correspondence should be addressed to A R Genazzani, Department of Reproductive Medicine and Child Development, Section of Gynecology and \\ Obstetrics, University of Pisa, Via Roma 35, 56100, Pisa, Italy; Email a.genazzani@obgyn.med.unipi.it)
}

\begin{abstract}
Objective: Neurosteroids have been suggested to be involved in the regulation of cognitive performances. A major neurosteroid gamma-aminobutyric acid (GABA) agonist is allopregnanolone: the main source of circulating allopregnanolone is the adrenal cortex. Studies indicated that a disturbance of the central regulation of the hypothalamic-pituitary-adrenocortical axis occurs in both senile (Alzheimer's disease: $\mathrm{AD}$ ) and vascular dementia (VD).

Design: The aim of the present study was to evaluate the levels of circulating allopregnanolone, dehydroepiandrosterone (DHEA) and cortisol and their response to corticotropin-releasing factor (CRF) test in $\mathrm{AD}$ and $\mathrm{VD}$.

Methods: Three groups of 12 subjects were included in the study: AD, VD and age-matched control subjects. CRF test was performed in all subjects and allopregnanolone, DHEA and cortisol levels were measured every $15 \mathrm{~min}$ for $2 \mathrm{~h}$.

Results: Mean \pm S.E.M. allopregnanolone and DHEA basal levels were significantly lower in AD and VD than in controls, while cortisol levels were significantly higher than in controls $(P<0.01)$. Allopregnanolone and DHEA levels increase in response to CRF test in all subjects but the area under curve (AUC) in patients was significantly lower than in controls $(P<0.01)$. Cortisol secretion appeared to be very sensitive in response to CRF stimulation: in fact, cortisol response to CRF test in AD and VD subjects was higher (both as AUC and as \% max increase) than in controls $(P<0.01)$.

Conclusions: The present study firstly showed that allopregnanolone levels are reduced both in $\mathrm{AD}$ and in VD and that dementia has a preserved stimulated response of allopregnanolone to CRF. Overall, however, the total response of allopregnanolone to CRF remains reduced in respect to controls. Further studies are necessary for a better understanding of the role of neurosteroids in the regulation of cognitive function.
\end{abstract}

European Journal of Endocrinology 142 466-471

\section{Introduction}

An increased vulnerability of neurons in Alzheimer's disease $(\mathrm{AD})$ is hypothesized to result from an imbalance of excitatory and inhibitory inputs. An involvement of the gamma-aminobutyric acid (GABA)ergic system in Alzheimer-related neuropathological changes has been demonstrated $(1,2)$. In particular, different benzodiazepine receptor agonists or antagonists are able to influence cognitive function by modulating GABAergic function in the brain (3-5). Recently, the brain was shown to be the source of de novo synthesized steroid hormones, named neurosteroids. Some of these bind to GABA A receptors and seem to be involved in modulating stress, cognitive performances and, in particular, in aggressive behavior of AD (6).

Allopregnanolone is one neuroactive steroid which acts as a GABA A receptor agonist, modulating behaviors, stress and neuroendocrine functions in rats. The increased synthesis of allopregnanolone in rat brain following acute stress suggests that this steroid may play a role as an endogenous stress-protective compound (7-9). Pretreatment with high doses of allopregnanolone significantly decreased corticotropinreleasing factor (CRF)-induced behavioral manifestations of stress and anxiety, indicating an anxiolytic, 
sedative-hypnotic and anti-aggressive effect similar to that produced by benzodiazepines (9). Conversely, dehydroepiandrosterone (DHEA) and its sulfated conjugated metabolite (DHEAS), both acting as GABA A antagonist neuroactive steroids, are able to improve well-being and cognitive function in aged subjects (10). While it is known that serum DHEA and DHEAS are reduced in $\operatorname{AD}(11,12)$, no data are available concerning allopregnanolone circulating levels.

Previous studies showed a disturbance in the central regulation of the hypothalamic-pituitary-adrenocortical axis (HPA) in both senile and vascular dementia (VD). Some behavioral disturbances in dementia appear as a consequence of an increased stress-induced HPA activity. In fact, these patients fail to adapt to chronic stress owing to an insufficient feedback system $(13,14)$. CRF appears as the key neuropeptide in the interplay between stress and cognition (15). Conflicting data are present in the literature concerning CRF levels in cerebrospinal fluid of AD patients (16-19), while a dramatic reduction in $\mathrm{CRF}$ concentration and an increase in $\mathrm{CRF}$ receptor density in the brain cortex have been described $(20,21)$. Cortisol response to CRF test has been used in $\mathrm{AD}$ patients to clarify the activity of HPA axis, with conflicting results $(16,22)$. CRF increases serum allopregnanolone in healthy subjects (23) while no data are available on allopregnanolone and DHEA response to CRF test in patients suffering from $\mathrm{AD}$ or VD. The aim of the present study was to evaluate circulating allopregnanolone, DHEA and cortisol basal levels and their response to CRF test in $\mathrm{AD}$ and VD.

\section{Materials and methods}

\section{Subjects}

The present study included three groups of subjects $(n=36)$ : group a, patients suffering from $\operatorname{AD}(n=12$ : 5 men and 7 women, age range $64-84)$; group b, patients suffering from VD $(n=12: 6$ men and 6 women, age range 65-82); and group c, control subjects $(n=12: 4$ men and 8 women, age range 68-81).

\section{Protocol}

Before entering the study each subject had medical history, physical examination and routine laboratory tests performed. Subjects with a history of cancer were excluded. None of the subjects were taking psychoactive medications, hormonal drugs, mineral or vitamin supplements or anti-inflammatory drugs. In group c, a diagnostic interview did not show current or recent (within the past 2 years) medical or psychiatric illness nor pathological changes in mood and behavior in recent years. In group a, AD was diagnosed by two independent psychiatrists according to the criteria of the Diagnostic and Statistical Manual of Mental Disorders IV (DSM IV). Patients with dementia of vascular origin (history of stroke, insufficiency of cerebral arteries, infatuate cerebral areas seen in computed tomography) were included in group b. After approval of the Local Ethical Committee, informed consent was obtained from each subject or from relatives after full description of the protocol.

In all patients a catheter was inserted into the antecubital vein and a slow infusion of $0.9 \%$ saline solution was started. A basal sample was drawn at time $-30 \mathrm{~min}$ before beginning the CRF test. The CRF test consisted of an intravenous bolus injection of $100 \mu \mathrm{g}$ hCRF (Clinalfa AG, Laufelfingen, Switzerland) and blood samples were taken at $0,15,30,45,60$ and 90 min after injection of CRF. Blood samples were centrifuged and serum stored at $-20{ }^{\circ} \mathrm{C}$ until assayed. Allopregnanolone, DHEA and cortisol were evaluated in each sample.

\section{Methods}

Allopregnanolone assay Analytical grade solvents were purchased from Merck (Darmstadt, Germany); C18 Sep-Pak cartridges were obtained from Waters Corporation (Milford, CT, USA). Standard allopregnanolone was purchased from Sigma Chemical Co. (St Louis, MO, USA) and pregnan-3 $\alpha$-ol-20-one, $5 \alpha$ - $[9,11,12,-$ $3 \mathrm{H}(\mathrm{N})]$ ( $45 \mathrm{Ci} / \mathrm{nmol}$ ) from Amersham (Amersham, Bucks, UK). The polyclonal antisera, raised in sheep against allopregnanolone carboxymethyl ether coupled to BSA, were kindly provided by Dr R H Purdy. Serum samples $(1 \mathrm{ml})$ were thawed. The assay was performed as previously described (23). The sensitivity of the assay, expressed as a minimal amount of allopregnanolone distinguishable from the zero sample with 95\% probability, was $15-20 \mathrm{pg} /$ tube and the intra- and interassay coefficients of variation were $7.2 \%$ and $9.1 \%$ respectively.

DHEA assay Serum samples for the determination of DHEA were extracted with ether, purified through a C18 Sep-Pak cartridge and then assayed by RIA using a trade kit (Radim SpA, Pomezia, Italy); the sensitivity was $15 \mathrm{pg} / \mathrm{ml}$ and the intra- and interassay coefficients of variation were $3.1 \%$ and $6.9 \%$ respectively.

Cortisol assay Cortisol was assayed using RIA methods (Radim SpA). The sensitivity of the method was $0.9 \mathrm{nmol} / \mathrm{l}$ and the intra- and interassay coefficients of variation were $3.6 \%$ and $7.3 \%$ respectively.

\section{Statistics}

The statistical analysis of the results was performed with a Macintosh personal computer using Abacus Concepts, Stat-View 4.0 program. All results are reported as the mean \pm s.E.M. Related measures ANOVA was used for comparison of hormone levels between times and Scheffé F-test was used to determine the presence of significant differences in mean values. 

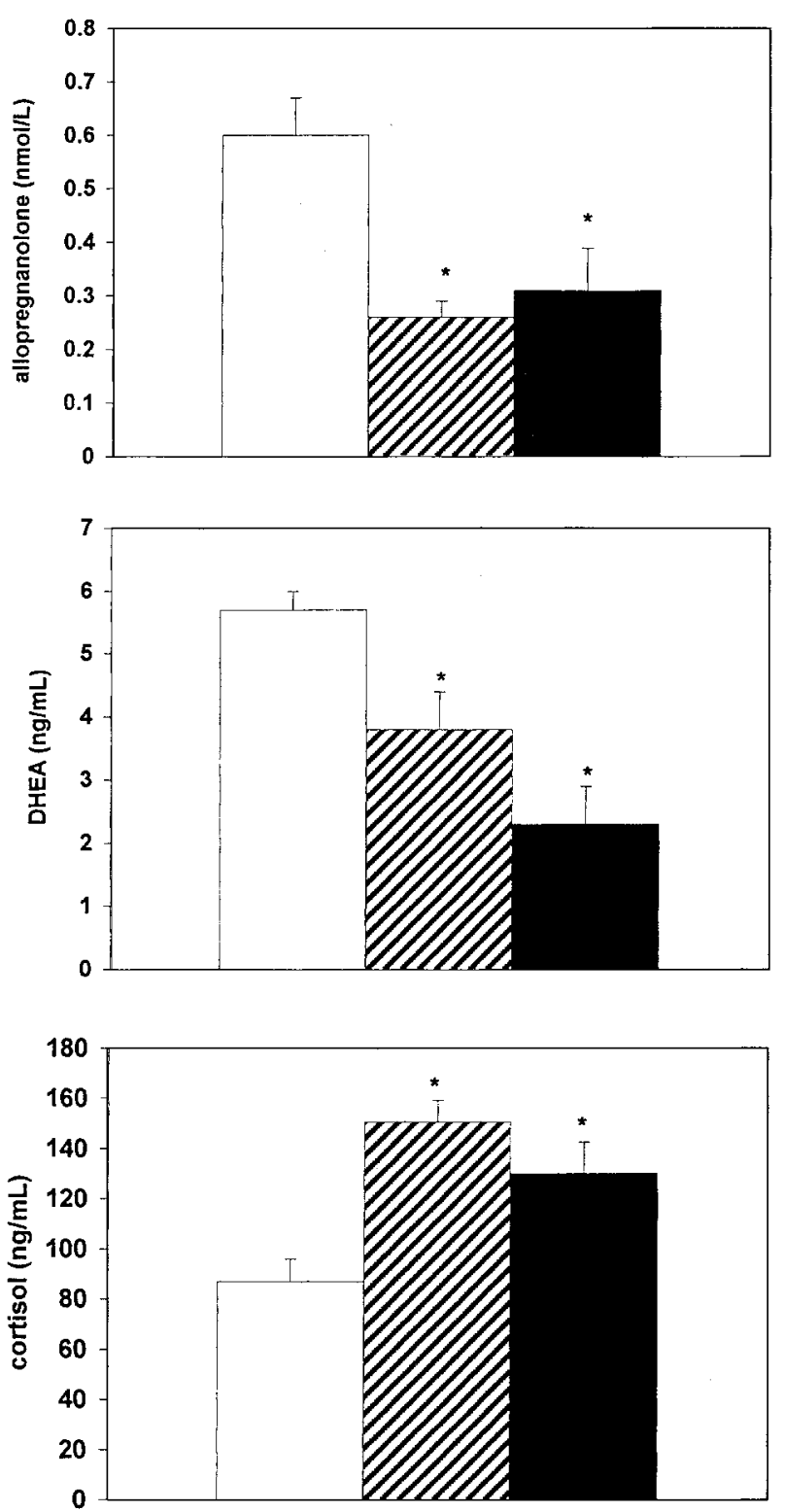

Figure 1 Mean \pm S.E.M. allopregnanolone, DHEA and cortisol basal levels. White column, controls; striped column, VD; and black column, AD. ${ }^{*} P<0.001$

\section{Results}

Patients suffering from AD or VD showed serum allopregnanolone and DHEA basal levels significantly lower than controls $(P<0.001)$, while cortisol levels were significantly higher $(P<0.001)$ (Fig. 1). Mean \pm S.E.M. allopregnanolone and DHEA levels increased in response to CRF test (Fig. 2) but the area under curve (AUC) in patients with AD or VD was significantly lower than in controls (Fig. 3) $(P<0.001)$. Serum cortisol increased in response to CRF (Fig. 2), and the AUC in AD and VD patients was significantly higher than in controls (Fig. 3). No significant difference either in
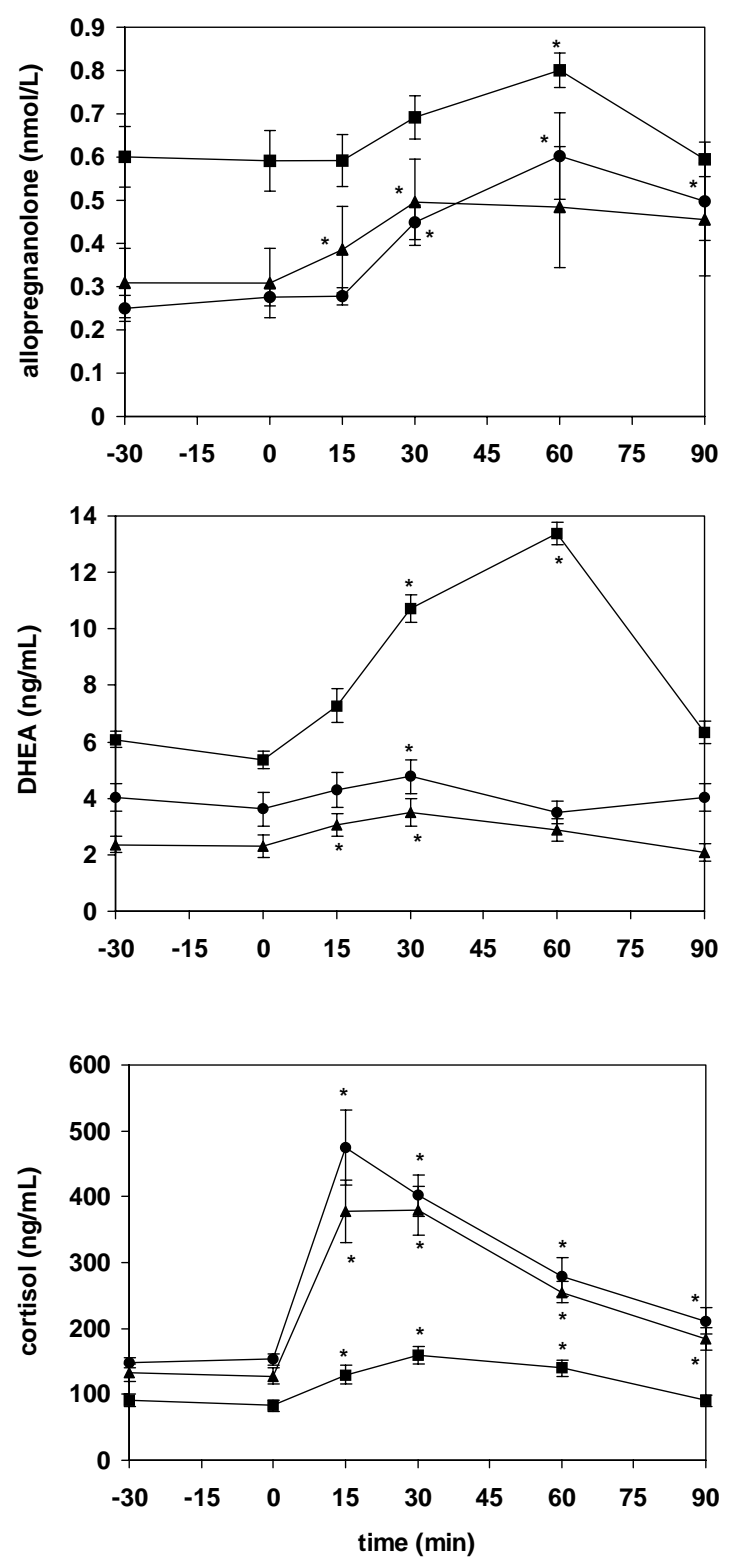

Figure 2 Mean \pm S.E.M. allopregnanolone, DHEA and cortisol response to CRF test in control $(\mathbf{\square}), A D(\mathbf{\Lambda})$ and VD $(\bullet)$ subjects. ${ }^{*} P<0.001$.

basal levels or in response to CRF test between patients suffering from $\mathrm{AD}$ or VD was observed.

\section{Discussion}

The present study firstly showed that $\mathrm{AD}$ and VD patients have low serum allopregnanolone levels compared with age-matched healthy controls, confirming that serum DHEA levels are low in dementia (11, 12 ). It is known that DHEA and DHEAS improve longterm memory and diminish amnesia in mice and enhance neuronal and glial survival and differentiation in cultures of embryonic mouse brain cells (24). The 

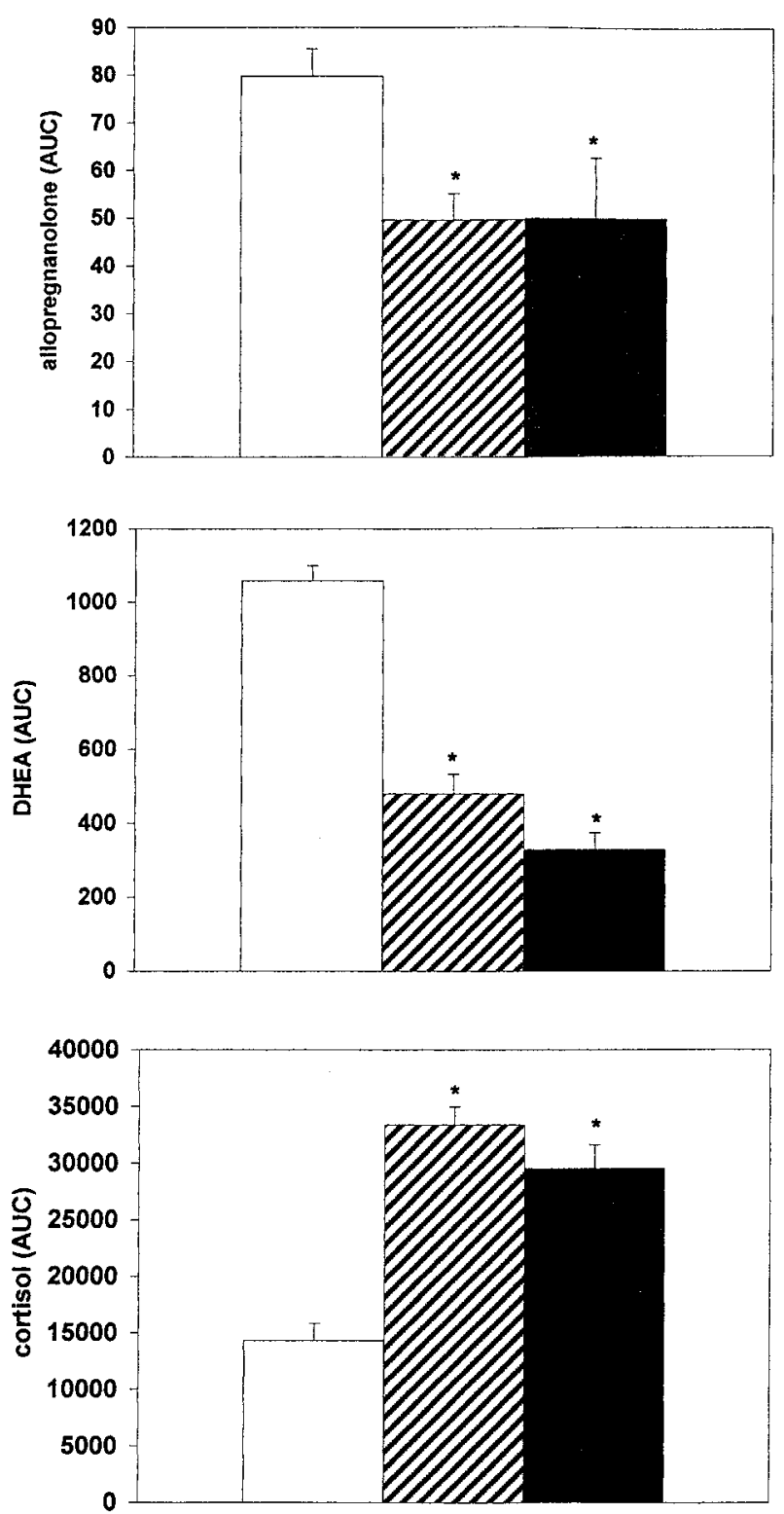

Figure 3 AUC of allopregnanolone, DHEA and cortisol response to $\mathrm{CRF}$ test. White column, controls; striped column, VD; and black column, AD. ${ }^{\star} P<0.001$.

administration of DHEA improves physical and psychological well-being and cognitive performances in aged subjects (10). The mechanism(s) of DHEA-mediated neuroprotection is unclear: it is probable that DHEA antagonizes some deleterious effects of cortisol which causes a progressive hippocampal damage and cognitive impairment in dementia (25-29). A low DHEA/cortisol ratio may be deleterious to hippocampal function (22). The hippocampus has a leading role in HPA axis regulation, but it is also an important brain site in memorization processing and in the connections between intellectual capacity and psychoaffective conditions; moreover, it is vulnerable to ipoxic-anoxic stimuli.
Experimental data in rats and primates showed that the increase in the glucocorticoid concentrations determines loss of specific receptors and neural damage in the hippocampus. Patients that underwent prolonged and high dose corticosteroid treatments showed memory and logical capacity deficits. It can be hypothesized that functional alterations of hippocampus glucocorticoid receptors for the negative feedback leads to a hyperactive HPA axis function, which in turn may cause further neural damage in the hippocampus; this could explain the correlation between cortisol levels and degree of brain vascular aging.

The finding that cortisol levels in $\mathrm{AD}$ and $\mathrm{VD}$ are higher than in control patients is in agreement with most of the literature $(13,22,30-32)$ and a correlation between the severity of dementia and the increase of cortisol levels has been reported $(12,13,33,34)$. Nevertheless, this is the first paper describing a reduction in allopregnanolone circulating levels in patients affected by $\mathrm{AD}$ or VD. The hypothesis that allopregnanolone might be involved in modulating cognitive function is supported by the evidence that conditions characterized by modifications in behavior, mood and cognitive performance, such as menstrual cycle, pregnancy and aging, are associated with changes in allopregnanolone levels $(23,35,36)$. It is possible to hypothesize that in dementia the low allopregnanolone levels are a consequence of the low CRF levels (23) that may explain also the low DHEA levels observed. On the other hand, both the AUC of DHEA and allopregnanolone response to CRF were reduced in dementia, indicating that these patients had an impairment in the total neuroendocrine balance capacity involving DHEA and allopregnanolone secretion. The different pattern observed between allopregnanolone and DHEA could reflect different metabolic pathways involved in their secretion and could indicate a major or more precocious involvement of DHEA secretion in dementia with respect to allopregnanolone.

The present study indicates that cortisol secretion is ultra-sensitive in response to CRF stimulation: in fact, cortisol response to CRF test was higher, both as AUC and as percentage maximum increase in $\mathrm{AD}$ and $\mathrm{VD}$ subjects, than in controls. Cortisol response to CRF test in $\mathrm{AD}$ have been described as similar $(16,37)$ or lower (30) than in controls; however, recent data are consistent with our findings of a higher cortisol response to $\mathrm{CRF}$ test (22) and adrenocorticotropin (ACTH) test (38) in $\mathrm{AD}$ patients. On the other hand, Dodt et al. (39) described a similar response of DHEA to CRF test in elderly and young subjects, regardless of their mental state; however, the simultaneous administration of vasopressin in association with CRF (half dose compared with the present study) means that the results are not comparable. The reason for the difference between cortisol and DHEA basal and stimulated levels in dementia remains unclear. The present study showed that in dementia an impairment of the DHEA/cortisol 
ratio appeared more clearly in response to CRF test than at basal levels, indicating that in dementia, i.e. the reverse of the situation seen in controls, CRF determined a huge stimulation of cortisol secretion and an abatement of DHEA release. Since the adrenal androgen-stimulating factor and the alterations in adrenal androgen synthesis have been proposed for explaining the enhanced cortisol and reduced DHEA secretion in the elderly (39), such factors might play a role also in dementia.

In addition, the present study demonstrates that the kind of dementia did not influence either allopregnanolone, DHEA and cortisol levels or their response to CRF. Previous evidence regarding the differences in neuroendocrine function between $\mathrm{AD}$ and VD patients indicated contrasting results: while neuroendocrine regulation of growth hormone $(\mathrm{GH})$ appeared more damaged in AD than in multi-infarct dementia patients (40), both $\mathrm{AD}$ and VD patients showed a similar behavior of plasma $\beta$-endorphin and cortisol circadian rhythms and similar abnormalities of dexamethasone suppression test $(41,42)$.

In conclusion, the present study firstly showed that serum allopregnanolone and DHEA levels are reduced both in $\mathrm{AD}$ and in $\mathrm{VD}$ and that allopregnanolone but not DHEA stimulated response to pharmacological doses of CRF test is maintained, although the low basal levels and the lower AUC showed an impairment of neuroendocrine balance involving allopregnanolone and DHEA in AD and VD patients. The present evidence supports the concept that dementia per se, independent of the nature, may be the result of an altered stress response, and the modifications in the circulating levels of allopregnanolone, a stress-related steroid hormone, might enter in this view. Therefore, it will be of interest to study the role of allopregnanolone in the complex way leading from stress to cognition, possibly via the interplay between brain CRF and CRF-related peptides.

\section{Acknowledgements}

This work was supported by the grant of Progetto Finalizzato Ministero della Sanità IRCCS 1995 RF 95239 'La Sindrome di Alzheimer: valutazioni neuroendocrine'.

\section{References}

1 Mizukami K, Ikonomovic MD, Grayson DR, Rubin RT, Warde D, Sheffield R, Hamilton RL, Davies P \& Armstrong DM. Immunohistochemical study of $\mathrm{GABA}(\mathrm{A})$ receptor beta $2 / 3$ subunits in the hippocampal formation of aged brains with Alzheimer-related neuropathological changes. Experimental Neurology 1997147 333-345.

2 Marczynski TJ. GABAergic deafferentation hypothesis of brain aging and Alzheimer's disease revisited. Brain Research Bulletin $199845341-379$.

3 Kawasaki K, Eigyo M, Ikeda M, Kihara T, Koike K, Matsushita A, Murata S, Shiomi T, Takada S \& Yasui M. A novel benzodiazepine inverse agonist, S-8510, as a cognitive enhancer. Progress in
Neuropsychopharmacology and Biological Psychiatry 199620 1413-1425.

4 Marczynski TJ. GABAergic deafferentation hypothesis of brain aging and Alzheimer's disease pharmacologic profile of the benzodiazepine antagonist, flumazenil. Review of Neuroscience $19956221-258$.

5 Polc P. Involvement of endogenous benzodiazepine receptor ligands in brain disorders: therapeutic potential for benzodiazepine antagonists? Medical Hypotheses 199544 439-446.

6 Pickles VR. Neurosteroids and aggressive behaviour in Alzheimer's disease. Medical Hypotheses 199442 243-244.

7 Barbaccia ML, Roscetti G, Trabucchi M, Concas A, Dazzi L, Purdy RI \& Biggio G. Brain allopregnanolone concentrations and GABA A receptor function in stressed rats, vol 21, pp 1345. New York: Society for Neuroscience 1995 Abstract 408.

8 Purdy RH, Morrow AL, Moore PH \& Paul 1991. Stress-induced elevations of $\gamma$-aminobutyric acid type A receptor-active steroids in the rat brain. Proceedings of the National Academy of Sciences of the USA 199188 4553-4557.

9 Patchev VK, Shoaib M, Holsboer F \& Almeida OFX. The neurosteroid tetrahydroprogesterone counteracts corticotropinreleasing hormone-induced anxiety and alters the release and gene expression of corticotropin-releasing hormone in the rat hypothalamus. Neuroscience 199462 265-271.

10 Morales AJ, Nolan JJ, Nelson JC \& Yen SSC. Effects of replacement dose of dehydroepiandrosterone in man and women of advancing age. Journal of Clinical Endocrinology and Metabolism 199478 $1360-1367$.

11 Nasman B, Olsson T, Backstrom T, Eriksson S, Grankvist K, Viitanen M \& Bucht G. Serum dehydroepiandrosterone sulfate in Alzheimer's disease and in multi-infarct dementia. Biological Psychiatry $199130684-690$.

12 Miller TP, Taylor J, Rogerson S, Mauricio M, Kennedy Q, Schatzberg A, Tinklenberg J \& Yesavage J. Cognitive and noncognitive symptoms in dementia patients: relationship to cortisol and dehydroepiandrosterone. International Psychogeriatrics $19981085-96$.

13 Gottfries CG, Balldin J, Blennow K, Brane G, Karlsson I, Regland B \& Wallin A. Hypothalamic dysfunction in dementia. Journal of Neural Transmission 1994 43 203-209.

14 Gottfries CG, Balldin J, Blennow K, Brane G, Karlsson I, Regland B \& Wallin A. Regulation of the hypothalamic-pituitary-adrenal axis in dementia disorders. Annals of the New York Academy of Science 1994746 336-343.

15 Lightman SL. Corticotropin-releasing factor. From stress to cognition. Nature 1995373 233-234.

16 Martignoni E, Petraglia F, Costa A, Bono G, Genazzani AR \& Nappi G. Dementia of the Alzheimer type and hypothalamus-pituitaryadrenocortical axis: changes in cerebrospinal fluid, corticotropin releasing factor and plasma cortisol levels. Acta Neurologica Scandinavica 199081 452-456.

17 Banki CM, Kamacsi L, Bissette G \& Nemeroff CB. Cerebrospinal fluid neuropeptides in mood disorder and dementia. Journal of Affective Disorders 199225 39-45.

18 Heilig M, Sjogren M, Blennow K, Ekman R \& Wallin A. Cerebrospinal fluid neuropeptides in Alzheimer's disease and vascular dementia. Biological Psychiatry 199538 210-216.

19 May C, Rapoport SI, Tomai TP, Chrousos GP \& Gold PW. Cerebrospinal fluid concentrations of corticotropin-releasing hormone $(\mathrm{CRH})$ and corticotropin $(\mathrm{ACTH})$ are reduced in patients with Alzheimer's disease. Neurology 198737 535-538.

20 De Souza EB, Whithehouse PJ, Kuhar MJ, Price DL \& Vale WV. Reciprocal changes in corticotropin-releasing factor (CRF)-like immunoreactivity and CRF receptors in cerebral cortex of Alzheimer's disease. Nature 1986319 593-595.

21 Behan DP, Khongsaly O, Owens MJ, Chung HD, Nemeroff CB \& De Souza EB. Corticotropin-releasing factor (CRF), CRF-binding protein (CRF-BP), and CRF/CRF-BP complex in Alzheimer's disease and control postmortem human brain. Journal of Neurochemistry 199768 2053-2060. 
22 Nasman B, Olsson T, Fagerlund M, Eriksson S, Viitanen M \& Carlstrom K. Blunted adrenocorticotropin and increased adrenal steroid response to human corticotropin-releasing hormone in Alzheimer's disease. Biological Psychiatry 199639 311-318.

23 Genazzani AR, Petraglia F, Bernardi F, Casarosa E, Tonetti A, Nappi RE, Luisi S, Palumbo M, Purdy RH \& Luisi M. Circulating levels of allopregnanolone in humans: gender, age and endocrine influences. Journal of Clinical Endocrinology and Metabolism 1998 83 2099-2103.

24 Roberts E. Dehydroepiandrosterone (DHEA) and its sulphate (DHEAS) as neural facilitators: effects on brain tissue in culture and on memory in young and old mice: a cyclic GMP hypothesis of action of DHEA and DHEAS in nervous system and other tissues. In The Biologic Role of Dehydroepiandrosterone, pp 13-42. Eds M Kalimi \& W Regelson. Berlin: Walter de Gruyter, 1990.

25 Sapolsky RM, Krey LC \& McEwen BS. The neuroendocrinology of stress and aging: the glucocorticoid cascade hypothesis. Endocrine Reviews 19867 284-301.

26 Wolkowitz OM, Reus VI, Manfredi F \& Roberts E. Antiglucocorticoid effects in Alzheimer's disease (reply). American Journal of Psychiatry 19921491126.

27 Wolkowitz OM, Reus VI \& Roberts E. Role of DHEA and DHEAS in Alzheimer's disease (reply). American Journal of Psychiatry 1993 1509.

28 Svec F \& Lopez A. Antiglucocorticoid actions of DHEA and low concentrations in Alzheimer's disease (letter). Lancet 19892 1335-1336.

29 Leblhuber F, Windhager E, Neubauer C, Weber J, Reisecker F \& Dienstl E. Antiglucocorticoid effects of DHEAS in Alzheimer's disease (letter). American Journal of Psychiatry $199130684-696$.

30 Hatzinger M, Z'Braun A, Hemmeter U, Seifritz E, Baumann F, Holsboer-Trachsler E \& Heuser IJ. Hypothalamic-pituitaryadrenal system function in patients with Alzheimer's disease. Neurobiology of Aging 199516 205-209.

31 Swanwick GR, Kirby M, Bruce I, Buggy F, Coen RF, Coakley D \& Lawlor BA. Hypothalamic-pituitary-adrenal axis dysfunction in Alzheimer's disease: lack of association between longitudinal and cross-sectional findings. American Journal of Psychiatry 1998155 286-289.

32 Hartmann A, Veldhius JD, Deuschle M, Standhardt H \& Heuser I. Twenty-four hour cortisol release profiles in patients with Alzheimer's and Parkinson's disease compared with normal controls: ultradian secretory pulsatility and diurnal variation. Neurobiology of Aging 199718 285-289.
33 Weiner MF, Vobach S, Olsson K, Svetlik D \& Risser RC. Cortisol secretion and Alzheimer's disease progression. Biological Psychiatry 199742 1030-1038.

34 Balldin J, Blennow K, Brane G, Gottfries CG, Karlsson I, Regland B \& Wallin A. Relationship between mental impairment and HPA axis activity in dementia disorders. Dementia $19945252-$ 256.

35 Majewska MD. Neurosteroids: endogenous bimodal modulators of the GABA-A receptor. Mechanism of action and physiological significance. Progress in Neurobiology 199238 379-395.

36 Schmidt PJ, Purdy RH, Moore PH, Paul SM \& Rubinow DR. Circulating levels of anxiolytic steroids in the luteal phase in women with premenstrual syndrome and in control subjects. Journal of Clinical Endocrinology and Metabolism 199479 12561260.

37 Martignoni E, Petraglia F, Costa A, Monzani A, Genazzani AR \& Nappi G. Cerebrospinal fluid corticotropin releasing factor levels and stimulation test in dementia of the Alzheimer type. Journal of Clinical and Laboratory Analysis 19904 5-8.

38 O'Brien JT, Ames D, Schweitzer I, Mastwyk M \& Colman P. Enhanced adrenal sensitivity to adrenocorticotrophic hormone (ACTH) is evidence of HPA axis hyperactivity in Alzheimer's disease. Psychological Medicine 199626 7-14.

39 Dodt C. Dittmann J. Hruby J, Spath-Schwalbe E. Born J. Schuttler $\mathrm{R} \&$ Fehm HL. Different regulation of adrenocorticotropin and cortisol secretion in young, mentally healthy elderly and patients with senile dementia of Alzheimer's type. Journal of Clinical Endocrinology and Metabolism 199172 272-276.

40 Higashi S, Fujita M, Nishimoto Y, Kuroda K, Asaba H \& Sakai T. Neuroendocrine studies in dementia patients: responses of plasma $\mathrm{GH}$ and PRL following bromocriptine administration. Acta Neurologica Scandinavica 199490 39-44.

41 Rolandi E, Gandolfo C, Franceschini R, Cataldi A, Garibaldi A \& Barreca T. Twenty-four-hour beta-endorphin secretory pattern in Alzheimer's disease. Neuropsychobiology 199225 188-192.

42 Balldin J, Gottfries CG, Karlsson I, Lindstedt G, Langstrom G \& Walinder J. Dexamethasone suppression test and serum prolactin in dementia disorders. British Journal of Psychiatry 1983143 277-281.

Received 29 September 1999

Accepted 29 December 1999 